\title{
Near-infrared spectroscopy for the detection and quantification of bacterial contaminations in pharmaceutical products
}

\author{
Cristina Quintelas ${ }^{a}$, Daniela P. Mesquita ${ }^{a}$, João A. Lopes ${ }^{b}$, Eugénio C. Ferreira $^{\mathrm{a}}$, \\ Clara Sousa, ${ }^{\mathrm{a}, *}$ \\ ${ }^{a}$ CEB-Centro de Engenharia Biológica, Universidade do Minho, Braga, Portugal \\ b iMed, Departamento de Farmácia Galénica e Tecnologia Farmacêutica, Faculdade de Farmácia, Universidade de Lisboa, Lisboa, Portugal
}

\section{A R T I C L E I N F O}

\section{Article history:}

Received 24 March 2015

Received in revised form 29 June 2015

Accepted 1 July 2015

Available online 4 July 2015

\section{Keywords:}

FT-NIRS

Bacterial contaminations

Pharmaceutical products

Chemometrics

Process analytical technology

\begin{abstract}
A B S T R A C T
Accurate detection and quantification of microbiological contaminations remains an issue mainly due the lack of rapid and precise analytical techniques. Standard methods are expensive and time-consuming being associated to high economic losses and public health threats. In the context of pharmaceutical industry, the development of fast analytical techniques able to overcome these limitations is crucial and spectroscopic techniques might constitute a reliable alternative. In this work we proved the ability of Fourier transform near infrared spectroscopy (FT-NIRS) to detect and quantify bacteria (Bacillus subtilis, Escherichia coli, Pseudomonas fluorescens, Salmonella enterica, Staphylococcus epidermidis) from 10 to $10^{8} \mathrm{CFUs} / \mathrm{mL}$ in sterile saline solutions ( $\mathrm{NaCl}$ 0.9\%). Partial least squares discriminant analysis (PLSDA) models showed that FT-NIRS was able to discriminate between sterile and contaminated solutions for all bacteria as well as to identify the contaminant bacteria. Partial least squares (PLS) models allowed bacterial quantification with limits of detection ranging from 5.1 to $9 \mathrm{CFU} / \mathrm{mL}$ for E. coli and B. subtilis, respectively. This methodology was successfully validated in three pharmaceutical preparations (contact lens solution, cough syrup and topic anti-inflammatory solution) proving that this technique possess a high potential to be routinely used for the detection and quantification of bacterial contaminations.
\end{abstract}

(c) 2015 Elsevier B.V. All rights reserved.

\section{Introduction}

Pharmaceuticals are widely used in a variety of ways in the prevention, treatment, and diagnosis of diseases. Although sterility is not a requirement in official compendia for non-sterile pharmaceuticals, bioburdens need to be within acceptable limits (Hugo and Russell, 1998). Accordingly with the European Pharmacopeia (European Pharmacopoeia, 2015), the presence of certain microorganisms in non-sterile preparations may have the potential to reduce or even inactivate the product's therapeutic activity and adversely affect patients' health. Any pharmaceutical product, whether manufactured in the hospital or industrial environment, has the potential to be contaminated with microorganisms, which may include bacteria, yeasts, or molds. Microbial contamination may be originated from raw materials, may be introduced during manufacture or contaminated during storage and use (Denyer and Baird, 2007). The occurrence of microbial

\footnotetext{
* Corresponding author at: Centro de Engenharia Biológica, Universidade do Minho, Campus de, Gualtar, 4710-057 Braga, Portugal.

E-mail address: clara.sousa@ceb.uminho.pt (C. Sousa).
}

contamination has been well documented, and contaminants range from true pathogens such as Clostridium tetani, to opportunistic ones like $P$. aeruginosa (Obuekwe et al., 2000). The European Pharmacopeia (European Pharmacopoeia, 2015) established the acceptance criteria for microbiological quality of nonsterile pharmaceuticals. These criteria impose the absence of different microorganisms, as E. coli, Staphylococcus aureus, P. aeruginosa, B. subtilis and Salmonella spp. and define limits to others. Standard methods for the quantification and identification of microorganisms in pharmaceuticals include fluorescent labeling (chemiluminescence) (Kricka, 1998; Roda and Guardigli, 2012), ATP bioluminescence (Denyer and Baird, 2007), chromatography and spectrophotometry (Sutton and Cundell, 2004), dye reduction, electrical resistance, enzyme monitoring (Denyer and Baird, 2007), Limulus amoebocyte lysate (Hussaini and Hassanali, 1987), nucleic acid probes (Sutton and Cundell, 2004; Anon., 2000) and phageinteraction technology (Denyer and Baird, 2007). Some of them are laborious, time-consuming and/or require expensive equipment and/or reagents. In this context, the development and implementation of methods for the identification and quantification of bacterial contaminations in pharmaceuticals able to overcome these limitations are imposed. 
Fourier transform near infrared spectroscopy (FT-NIRS) combined with chemometric methods is frequently chosen for process analytical technology as to obtain timely measurements of solids, liquids and other pharmaceutical forms (Bakeev, 2005). It is mainly used for its speed, low cost and non-destructive characteristics (Sarraguça and Lopes, 2009). This technique was already used for the detection and identification of bacteria in simple liquid suspensions (Rodriguez-Saona et al., 2004, 2001). However, and as far as authors are aware, it was not explored for the identification and quantification of bacteria in pharmaceutical products.

The aim of this work was to test the ability of FT-NIRS to detect and quantify bacterial contaminations in saline solutions $(\mathrm{NaCl}$ $0.9 \%$ ) and pharmaceutical preparations. Five different species usually responsible for microbial contamination of pharmaceutical products were used (B. subtilis, E. coli, P. fluorescens, S. enterica, and $S$. epidermidis). The methodology was successfully tested in saline solutions $(\mathrm{NaCl} 0.9 \%)$ and validated in three different pharmaceutical preparations (contact lens solution, cough syrup and topic anti-inflammatory solution).

a)

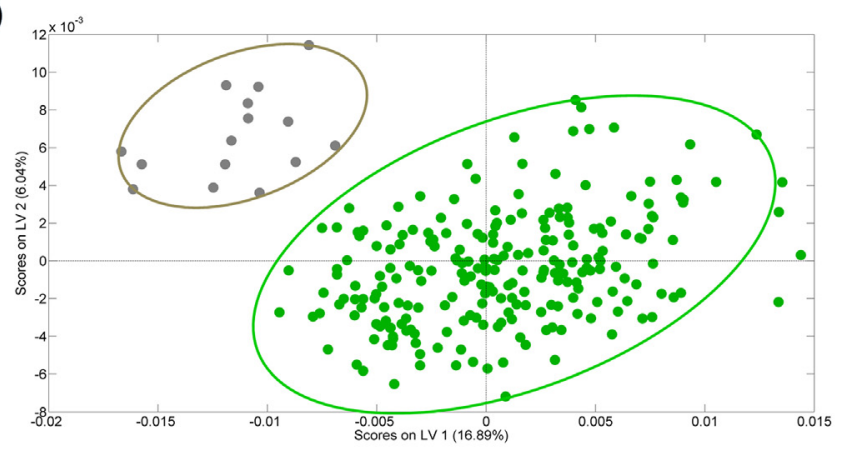

c)

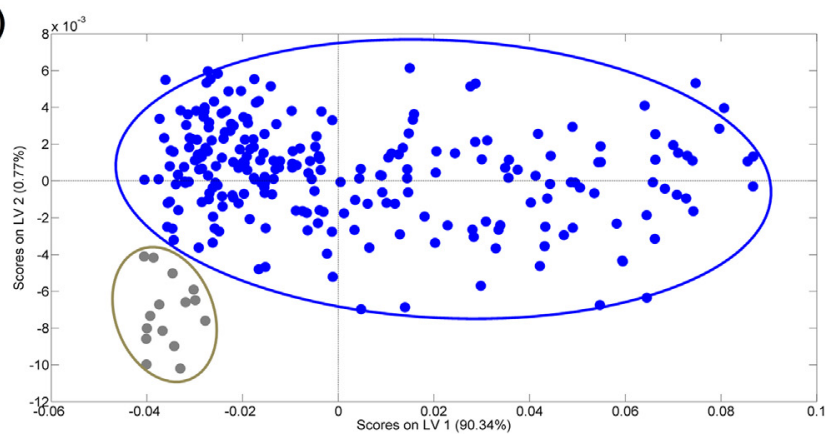

\section{Material and methods}

\subsection{Contaminated sample solutions}

Bacterial isolates used in this work (B. subtilis, E. coli, $P$. fluorescens, $S$. enterica and $S$. epidermidis) were grown from frozen stocks in tryptic soy agar $\left(37^{\circ} \mathrm{C} / 16 \mathrm{~h}\right)$ prior to the experiments. For each bacterium, a single colony was picked and grown in tryptic soy broth $\left(37^{\circ} \mathrm{C} / 16 \mathrm{~h}\right)$. Bacterial suspension was washed twice with a saline sterile solution. The pellets were re-suspended to an $\mathrm{OD}_{600 \mathrm{~nm}}=0.132$ and 16 contaminated saline solutions for each bacterium were prepared through serial dilutions (from 10 to $10^{8} \mathrm{CFU} / \mathrm{mL}$ ) and used in the proof-of-concept. A sterile saline solution was used as the negative control.

The pharmaceutical preparations used in this work were acquired in a local drug store: (a) contact lens solution-sodium chloride, boric acid, sodium tetraborate, sodium citrate, poloxamer 407, hyaluronic acid, disodium edetate $0.02 \%$, polyhexamethylene biguanide $0.0001 \%$ in purified water; (b) cough syrup-dextromethorphan $1.8 \mathrm{mg} / \mathrm{mL}$ and (3) topic anti-inflammatory solution- b)

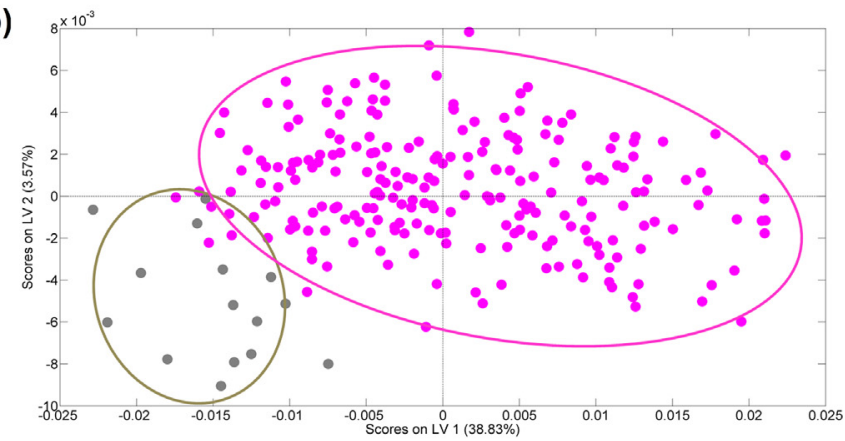

d)

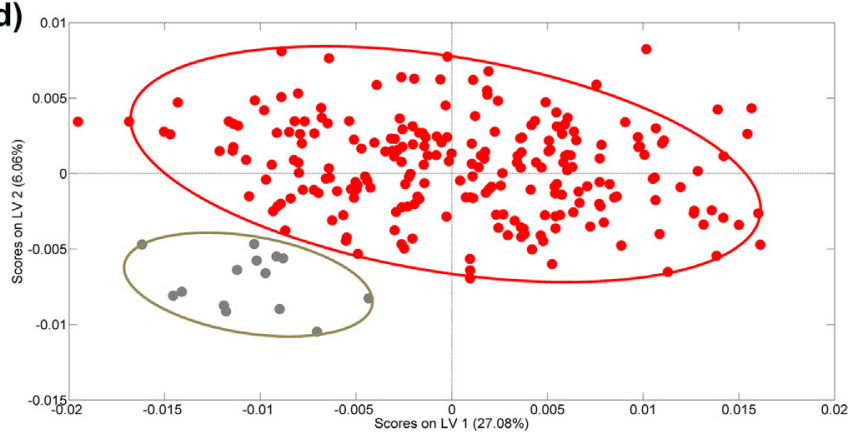

e)

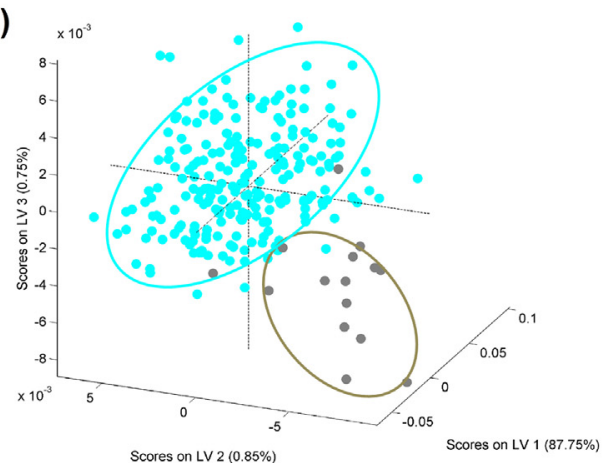

Fig. 1. Score plots of the PLSDA regression models in the spectral range $6000-5400 \mathrm{~cm}^{-1}$ of the discrimination between non-contaminated (๑) and contaminated saline

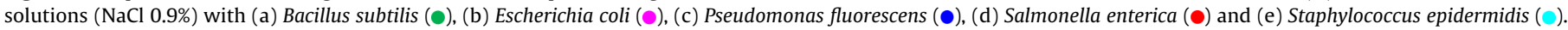



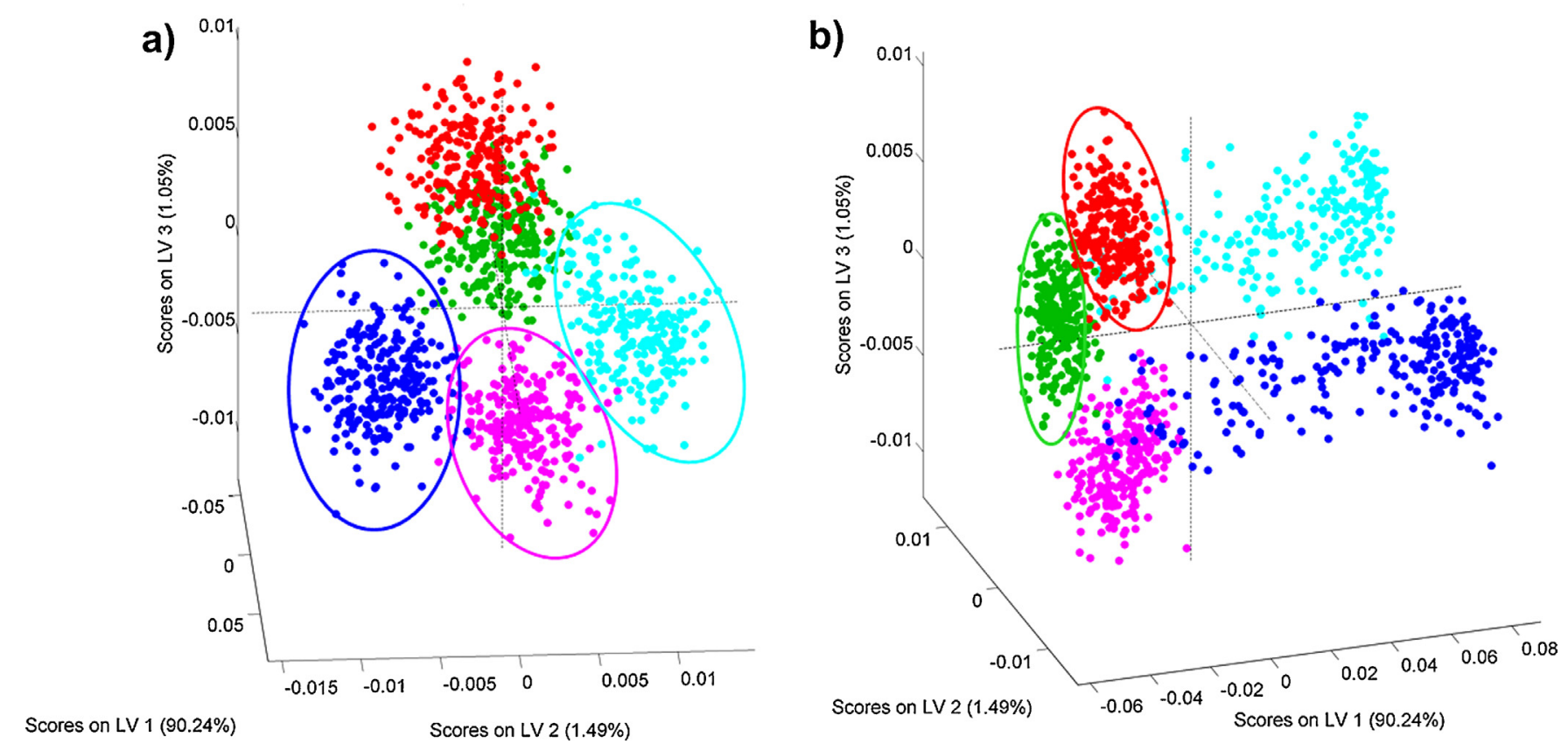

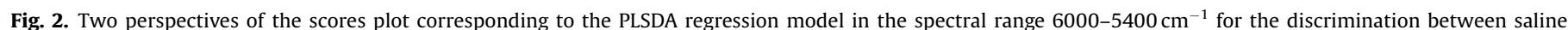

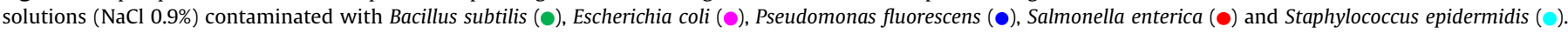

Table 1

Confusion matrix obtained for the the PLSDA species discrimination model, considering the region $6000-5400 \mathrm{~cm}^{-1}$ and $8 \mathrm{LV}$ (values are in $\%$ ).

\begin{tabular}{|c|c|c|c|c|c|c|}
\hline \multirow[t]{2}{*}{ FT-NIRS species prediction } & \multicolumn{6}{|l|}{ Species } \\
\hline & B. subtilis & E. coli & P. fluorescens & S. enterica & S. epidermidis & \\
\hline B. subtilis & 20.00 & 0 & 0 & 0.10 & 0.01 & - \\
\hline E. coli & 0 & 20.00 & 0 & 0 & 0 & - \\
\hline P. fluorescens & 0 & 0 & 20.00 & 0 & 0 & - \\
\hline S. enterica & 0 & 0 & 0 & 19.90 & 0 & - \\
\hline S. epidermidis & 0 & 0 & 0 & 0 & 19.99 & - \\
\hline Sum & 20.00 & 20.00 & 20.00 & 19.90 & 19.99 & 99.89 \\
\hline
\end{tabular}

indomethacin $10 \mathrm{mg} / \mathrm{g}$ ). Prior to the experiments, preparations were incubated over-night in tryptic soy broth followed by overnight incubation in tryptic soy agar to ensure its sterility. A contaminated saline solution of each bacterium was prepared as described above and used to contaminate the pharmaceutical preparations ( 16 serial dilutions, from 10 to $10^{8} \mathrm{CFU} / \mathrm{mL}$ ). For each solution (saline, pharmaceutical and controls) were obtained 10 congruent near-infrared spectra.

\subsection{Cell enumeration}

The exact number of bacterial cells in each contaminated saline solution (expressed in CFUs) was determined by using a standard classical counting method. Briefly, $100 \mu \mathrm{L}$ of the serial dilutions were incorporated in plate count agar medium, incubated $\left(37^{\circ} \mathrm{C}\right.$, $24 \mathrm{~h}$ ), and plates containing $30-300 \mathrm{CFUs}$ were selected for counting (Buchbinder et al., 1951). This procedure was performed in the contaminated saline solutions $(\mathrm{NaCl} 0.9 \%)$ used in the proofof-concept as well as in the solutions used to contaminate the pharmaceutical preparations. Details about the exact cellular concentration $(\mathrm{CFU} / \mathrm{mL})$ of each solution were provided in Table S1 of the Supplementary material.

\subsection{NIR experiments}

Near infrared spectra were recorded on a Fourier-transform near infrared spectrometer (FTLA 2000, ABB, Thermo Electron Corporation) equipped with an indium-gallium-arsenide (InGaAs) detector and connected to a transflectance probe (model FLEX-NIR$12 S-300 / 070504 / 2$, Sapphire Optics) with an optical path length of $2 \mathrm{~mm}$. The probe was connected through optical fibers to the NIR spectrometer and allowed measurements between 500 and $2500 \mathrm{~nm}$. For each sample, sixty-four scans were made and then averaged. The integration time was adjusted until the peaks at $1100-1200 \mathrm{~nm}$ for NIR were close to 60,000 intensity units. Grams/ AI software (Thermo Electron Corporation) was used for spectrometer configuration, control, and data acquisition. The background was taken every day from an empty probe ensuring that the probe is dry and cleaned. All solutions were measured by FT-NIRS at room temperature.

\subsection{Data analysis}

Near-infrared spectra were pre-processed with Savitzky-Golay filter (7 smoothing points, 2nd order polynomial and 2nd derivative) (Savitzky and Golay, 1964) to remove baseline drifts. 

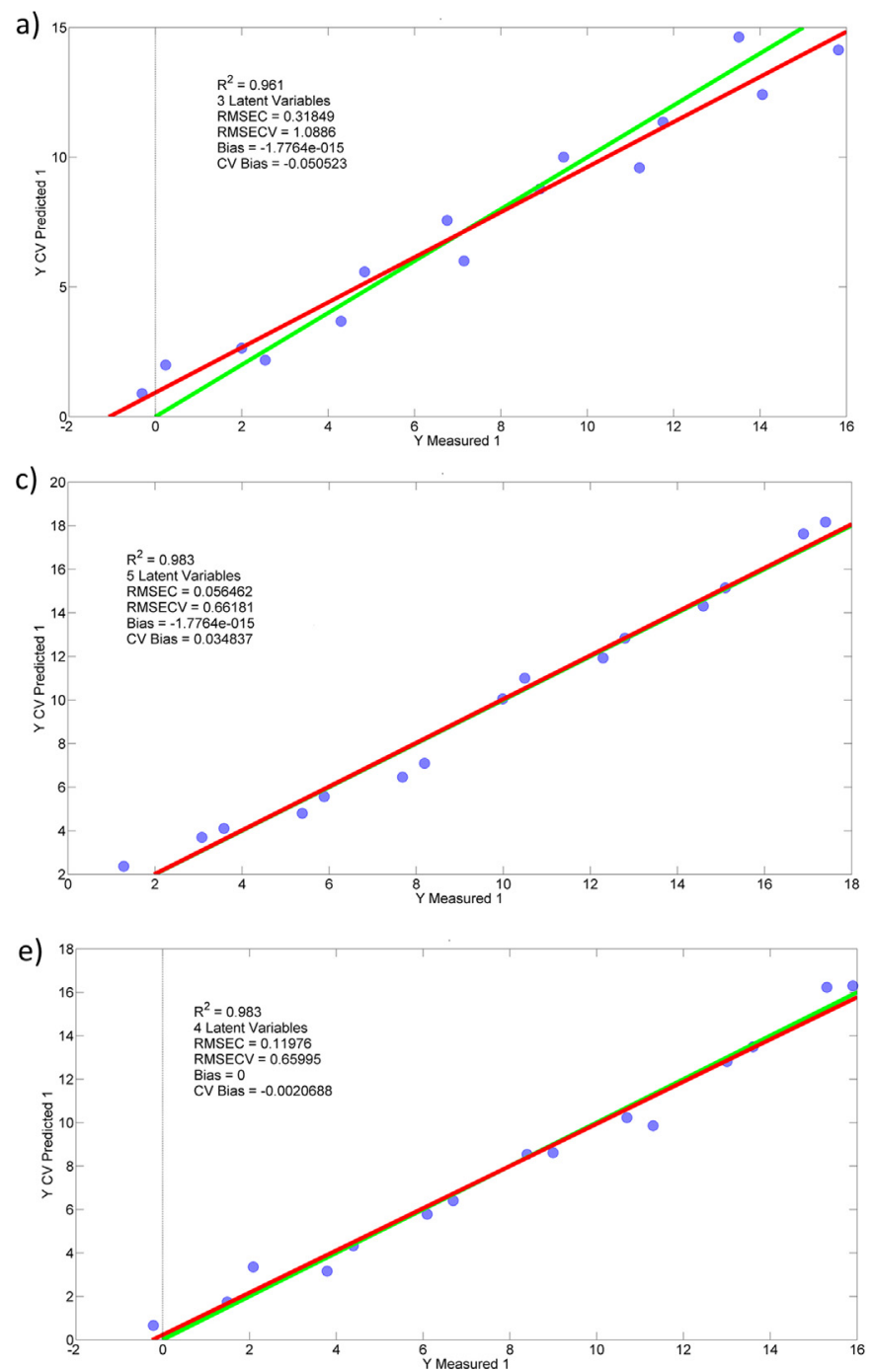
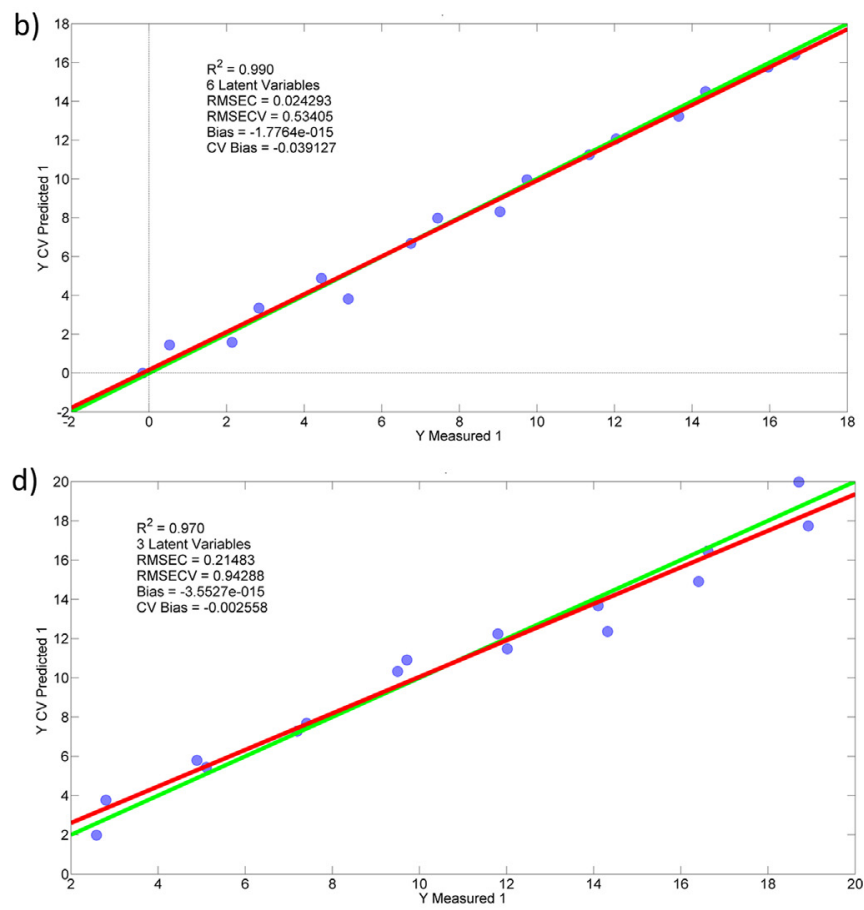

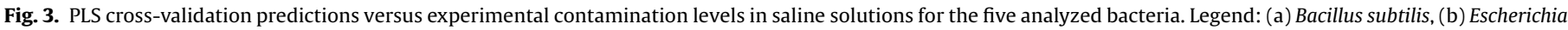
coli, (c) Pseudomonas fluorescens, (d) Salmonella enterica and (e) Staphylococcus epidermidis.

Table 2

Figures-of-merit of the PLS models developed for the sodium chloride solutions $0.9 \%(\mathrm{~m} / \mathrm{v})$ contaminated with Bacillus subtilis, Escherichia coli, Pseudomonas fluorescens, Salmonella enterica and Staphylococcus epidermidis.

\begin{tabular}{llllll}
\hline & No. LVs & $R^{2}$ & RMSEC & RMSECV & LOD (Alsberg et al., 1998) \\
\hline B. subtilis & 3 & 0.961 & 0.318 & 1.09 & 9.0 \\
E. coli & 6 & 0.990 & 0.0243 & 0.534 & 5.1 \\
P. fluorescens & 5 & 0.983 & 0.0565 & 0.662 & 5.7 \\
S. enterica & 3 & 0.970 & 0.215 & 0.943 & 7.8 \\
S. epidermidis & 4 & 0.983 & 0.120 & 0.660 & 5.7 \\
\hline
\end{tabular}

LV: latent variables; $R^{2}$ : correlation coefficient; RMSEC: root mean square error of the calibration; RMSECV: root mean square error of the cross validation; LOD: limit of detection ( $\mathrm{LOD}=3 \times e^{\mathrm{RMSECV}}, \mathrm{CFUs} / \mathrm{mL}$ ).

Spectra were cut to the analysis selected window and subjected to standard normal variate (SNV). Partial least squares (PLS) (Geladi and Kowalsky, 1986) and partial least squares discriminant analysis (PLSDA) (Barker and Rayens, 2003) were selected to model spectral data.

PLS regression models were used to estimate contamination levels (the PLS-1 algorithm was selected). Models were optimized for the number of latent variables (LVs) considering the leave-one- out cross-validation strategy. Prediction results are analyzed according to the root-mean-square-error of cross-validation (RMSECV) that is a measure of the model accuracy. The limit-ofdetection was estimated according Alsberg et al. (1998).

PLSDA is based on the PLS-2 algorithm (Geladi and Kowalsky, 1986; Barker and Rayens, 2003). PLSDA were applied to verify contamination and for bacteria discrimination purposes. The class decision criterion for PLSDA results was based on the maximum value obtained for each predicted class (Barker and Rayens, 2003). Models are developed on a fraction of the available number of samples (calibration set $-70 \%$ of the data) and tested on the remaining (prediction set $-30 \%$ of the data). This division is obtained randomly. The optimal number of latent variables for PLS is obtained by cross-validation (leave-one-out) using the calibration set. Discrimination results are expressed in the form of confusion matrices that compare the real assignment of each sample with the PLSDA model prediction for prediction set.

Before developing PLS or PLSDA models, datasets are meancentred (Geladi and Kowalsky, 1986). All chemometric models were performed in Matlab version 7.4 Release 2007a (MathWorks, Natick, MA) and PLS Toolbox version 4.2.1 for Matlab (Eigenvector Research, Manson, WA). 

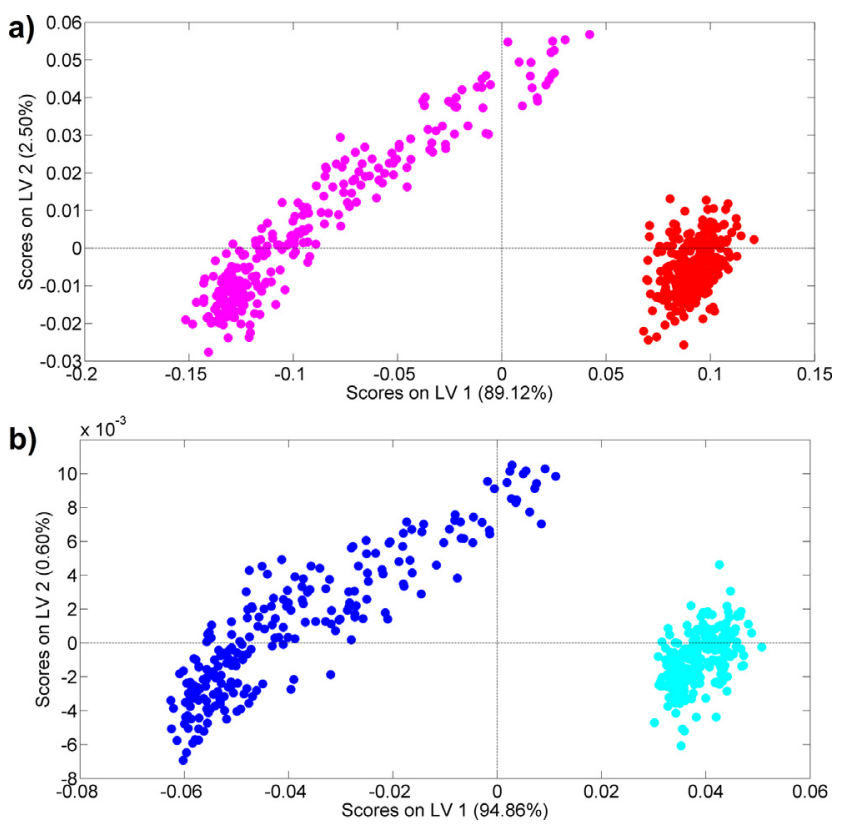

Fig. 4. Score plots of the PLSDA regression models in the spectral range 6000$5400 \mathrm{~cm}^{-1}$ of the discrimination between (a) cough syrup solutions contaminated with Escherichia coli ( $\bullet$ and Salmonella enterica (•), and (b) topic anti-inflammatory solutions contaminated with Pseudomonas fluorescens (@) and Staphylococcus epidermidis (๑).

\section{Results}

\subsection{Rationale of the study}

This study had a twofold objective: (I) to perform a proof-ofconcept in which we evaluate the ability of FT-NIRS coupled with chemometrics to detect and quantify bacterial contaminations in saline solutions $(\mathrm{NaCl} 0.9 \%)$, and (II) to test and validate this approach in several pharmaceutical preparations. The proof-ofconcept was developed with saline solutions contaminated with B. subtilis, E. coli, P. fluorescens, S. enterica and S. epidermidis (from 10 to $10^{8} \mathrm{CFUs} / \mathrm{mL}$ ). Firstly, we evaluated the ability of FT-NIRS to discriminate sterile from contaminated saline solutions independently of their cellular concentration for all the bacterial species included in this work. We then assess its potential identify the contaminating bacteria and the cellular concentration of the contaminated solutions. The developed methodology was then applied to three pharmaceutical preparations (contact lens solution, cough syrup and topic anti-inflammatory solution) to evaluate the potential of FT-NIRS to be routinely used as an alternative method to detect and quantify bacterial contaminations in water base pharmaceutical preparations. All procedures were repeated in different days to evaluate the reproducibility and repeatability of the technique.

\subsection{Proof-of-concept}

Near-infrared spectra obtained from saline solutions $(\mathrm{NaCl}$ $0.9 \%$ ) and pharmaceutical preparations (sterile or contaminated), present the typical shape of a near-infrared spectra showing large and broad bands (combinations and overtones) originating from $\mathrm{O}-\mathrm{H}$ in water. Due to the high water content of all samples, only the spectral region from 6000 to $5400 \mathrm{~cm}^{-1}$ was selected for chemometric analysis. The rationale is the exclusion of regions dominated by the absorption bands of water while keeping $\mathrm{C}-\mathrm{H}$ vibrations of alkanes, alkenes and aromatics.
For each bacterium, a partial least squares discriminant analysis (PLSDA) model was developed to assess the ability of FT-NIRS to discriminate the non-contaminated saline solution from contaminated ones (the entire range of concentrations were considered for the analysis-please see concentration details in Table S1 of Supplementary material). A clear discrimination between contaminated and non-contaminated solutions was achieved for all the bacteria included in this study as can be observed by analyzing the PLSDA model scores in Fig. 1. For all bacteria but one, a two component model was enough to discriminate solutions with only a small overlap observed in E. coli. (Fig. 1b). For S. epidermidis a three-component model was needed for the discrimination. Additionally, a PLSDA model was developed to evaluate the discrimination of contaminated saline solutions according to the contaminant bacteria. The scores plot of the PLSDA model (Fig. 2a and $b$ showing two different perspectives of the exactly same plot) clearly showed five distinct clusters. Each cluster corresponds to one bacterial species evidencing the ability of this spectroscopic technique for bacterial discrimination. The confusion matrix of the PLSDA model considering 8 latent variables (LV) (Table 1) yielded $99.9 \%$ of correct predictions being the worst case obtained for S. enterica with $99.5 \%$ of correct predictions. It is of note that $B$. subtilis, E. coli and $P$. fluorescens were always correctly predicted (100\%).

It is noteworthy that both discriminations (between contaminated and non-contaminated solutions and according to the contaminant bacteria) still occur at very low concentrations (about $10 \mathrm{CFU} / \mathrm{mL}$ ). The exact same procedure was repeated in different days space by two weeks and comparable results were achieved (data not shown).

For quantitative purposes, a partial least squares (PLS) model was developed for each contaminated saline solution considering samples with concentrations ranging from 10 to $10^{8} \mathrm{CFUs} / \mathrm{mL}$ (Fig. 3a-e). Results are summarized in Table 2. A good correlation $\left(R^{2}\right.$, Table 2$)$ was achieved being the worst case the saline solutions contaminated with B. subtilis. Both errors, obtained from calibration (RMSEC) and cross-validation (RMSECV), were found to be very satisfactory. Moreover, considering the error of crossvalidation and the concentrations range for each bacterium, it was possible to quantify, with errors lower than $5 \%$ : B. subtilis to a concentration of $73 \mathrm{CFU} / \mathrm{mL}$ (4\%), E. coli to $85 \mathrm{CFU} / \mathrm{mL}(2 \%)$, P. fluorescens to $36 \mathrm{CFU} / \mathrm{mL}$ (5\%), S. enterica to $133 \mathrm{CFU} / \mathrm{mL}(2 \%)$ and S. epidermidis to $44 \mathrm{CFU} / \mathrm{mL}$ (4\%). The method's results yielded very low limits of detection (LOD). In an independent day, the quantification procedure was repeated for the E. coli contaminated solutions and analogous results were obtained with a LOD of the same magnitude and a similar $R^{2}$ (data not shown).

\subsection{Pharmaceutical preparations}

We firstly proved the ability of FT-NIRS to detect and quantify bacterial contaminations in saline solutions. We further aimed to test this approach in real pharmaceutical preparations. In this context, three distinct preparations were selected (contact lens solution, cough syrup and topic anti-inflammatory solution) to strength the findings of our study. Similarly to the saline solutions, FT-NIRS was able to discriminate the non-contaminated pharmaceutical preparations from the contaminated ones for each bacterium independently of its concentration (data not shown). Moreover, it was also possible to discriminate contaminated cough syrup solutions with $E$. coli from those contaminated with S. enterica and the topic antiinflammatory solutions contaminated with $P$.fluorescens from those contaminated with $S$. epidermidis (Fig. $4 \mathrm{a}$ and b, respectively). The confusion matrix of the PLSDA models yielded a percentage of correct predictions for species discrimination of 100\% for both solutions (cough syrup and topic anti-inflammatory solution). 
a)

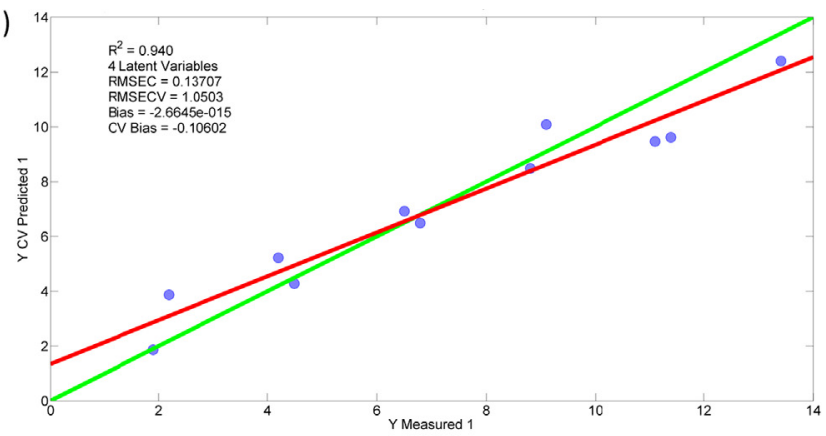

c)

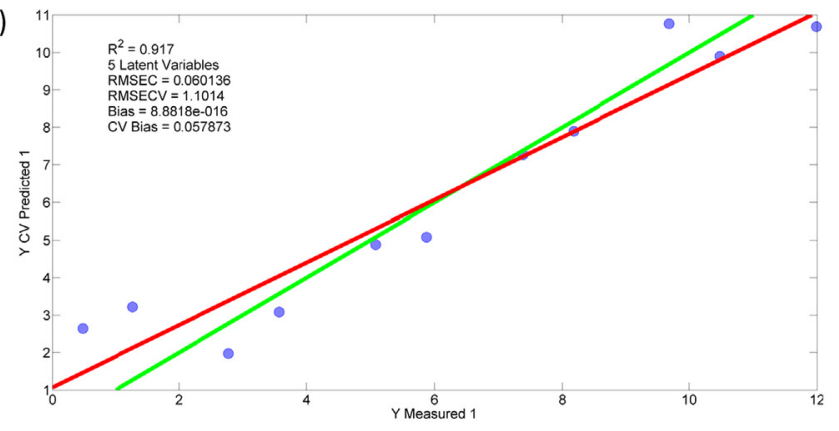

e)

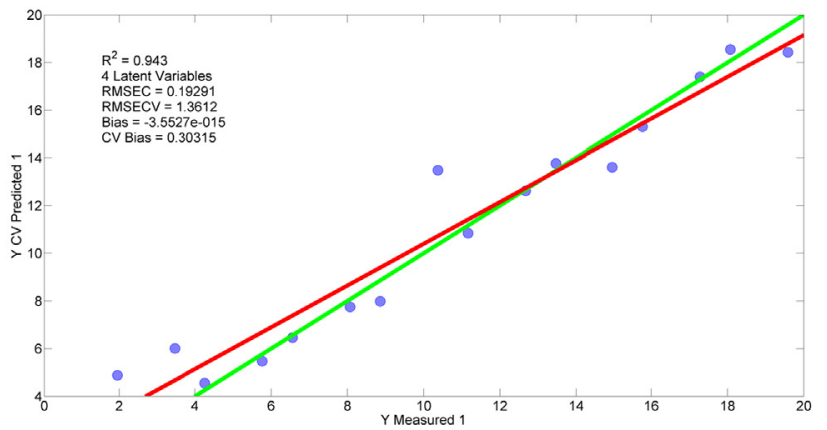

b)

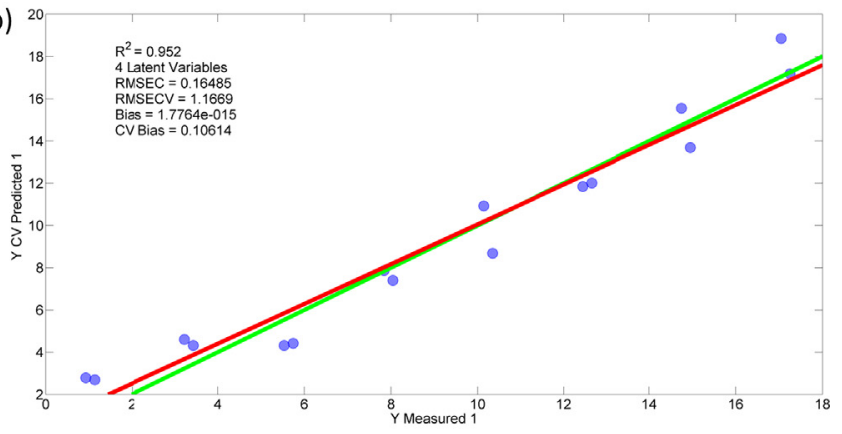

d)

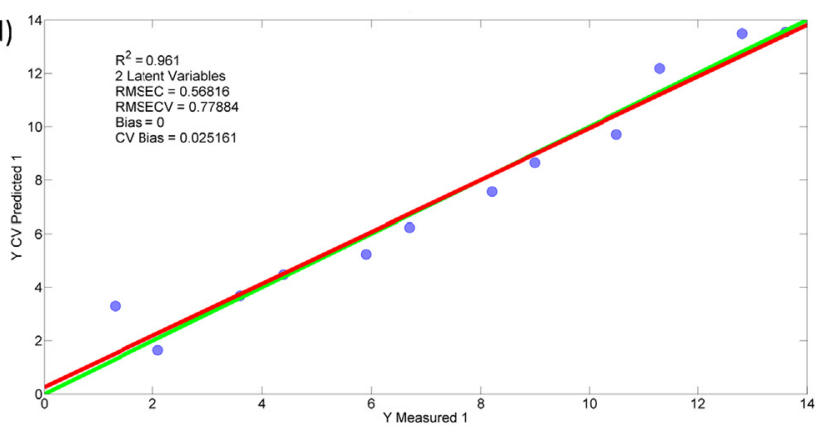

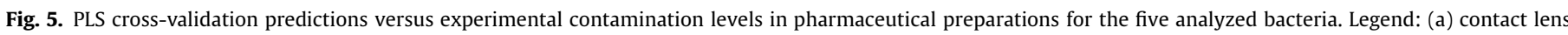

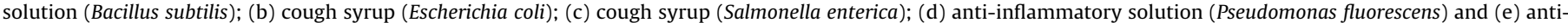
inflammatory solution (Staphylococcus epidermidis).

Table 3

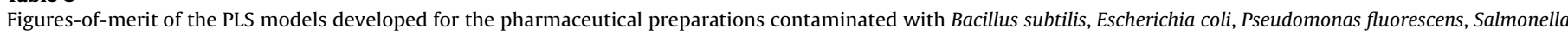
enterica and Staphylococcus epidermidis.

\begin{tabular}{|c|c|c|c|c|c|c|}
\hline Pharmaceutical preparation & Contaminant & No. LVs & $R^{2}$ & RMSEC & RMSECV & LOD (Alsberg et al., 1998) \\
\hline Contact lens solution & B. subtilis & 4 & 0.940 & 0.137 & 1.05 & 8.7 \\
\hline Cough syrup & E. coli & 4 & 0.952 & 0.165 & 1.17 & 9.6 \\
\hline Cough syrup & S. enterica & 5 & 0.917 & 0.0601 & 1.10 & 9.0 \\
\hline Anti-inflamatory & P. fluorescens & 2 & 0.961 & 0.568 & 0.779 & 6.6 \\
\hline Anti-inflammatory & S. epidermidis & 4 & 0.943 & 0.193 & 1.36 & 11.7 \\
\hline
\end{tabular}

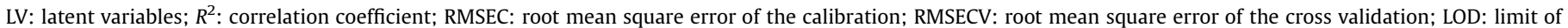
detection ( $\left.\mathrm{LOD}=3 \times e^{\mathrm{RMSECV}}, \mathrm{CFUs} / \mathrm{mL}\right)$.

Regarding the analysis achieved through the PLS models (Fig. 5, Table 3 ) it can be seen that it was also possible to quantify the cellular concentration for each bacteria in the pharmaceutical preparations with RMSEC and RMSECV very similar to those obtained for the saline solutions. Also, the LOD obtained was very satisfactory. In an independent day, a newly contact lens solution contaminated with $B$. subtilis was tested and the results obtained for the PLS model regarding LOD and $R^{2}$ were in accordance with initial results (data not shown).

\section{Discussion}

In this work we proved the ability of FT-NIRS to detect, discriminate and quantify microbiological contaminations in saline solutions ( $\mathrm{NaCl} 0.9 \%$ ). Moreover, using real pharmaceutical preparations, we have shown that this infrared based spectroscopic technique possesses a great potential to be used as an alternative technique for quality control in the pharmaceutical industry. FT-NIRS is a quick, low-cost and environmentally friendly technique with a 
very low analysis time that could be performed by lower skills personal making it very attractive for the industry. Several authors already addressed the potential of FT-NIRS for the pharmaceutical industry, essentially to monitor solid forms processes. Applications include the identification and quality diagnosis of raw-materials, active pharmaceutical ingredients and/or excipients quantification and monitoring/control of intermediate solid forms manufacturing process stages such as blending, granulation, tableting, coating and packing (Tomuta et al., 2013; Palou et al., 2012; Wahl et al., 2014; Blanco et al., 2008). Its ability to detect and quantify microbiological contamination was frequently explored in the context of food contaminations (Rodriguez-Saona et al., 2004, 2001; Tito et al., 2012) but barely explored in pharmaceutical contexts. To our knowledge this is the first time that a systematic procedure was tested and applied in several microorganisms and real pharmaceutical preparations with such promising results. In this study one tested the bacterial species most often responsible for microbiological contaminations in the pharmaceutical industry; however, other species could be detected individually by the same procedure. Also, the bacterial species select to include in this work possess distinct characteristics (gram type, coccus, and bacillus, true and opportunistic pathogens) to ensure that the procedure remains feasible independently of the contaminant bacteria. It is of note that this methodology could also be used for multiple bacterial species once it seems to be able to detect whether a solution is or not contaminated and to quantify the total number of pathogens independently of the bacterial species present in the solution. However, if the identification of the species present in the solution as contaminant is needed new PLSDA models should be developed and optimized with two or more pathogens for correct species identification. The European Pharmacopoeia 8.2 (in Microbiological quality of non-sterile products for pharmaceutical use) establishes the acceptance criteria for the microbiological quality of non-sterile dosage forms pointing the tolerable total aerobic microbial count (TAMC) (European Pharmacopoeia, 2015). In the case of oral aqueous solutions and cutaneous or nasal preparations, which is the case of the hereby tested pharmaceutical preparations (except contact lens solution), the allowed TAMC is $10^{2} \mathrm{CFU} / \mathrm{mL}$ (meaning a maximum acceptable count of 200). Despite the TAMC, for certain pharmaceutical preparations, the absence of specific microorganisms should be guaranteed. Our results clearly fit the acceptance criteria established in the European Pharmacopoeia 8.2 stressing the potential of this spectroscopic technique. Additionally, the replicates performed pointed to the possibility of introducing this method and comply with reproducibility and repeatability analytical method criteria. It is of note that FT-NIRS is an infrared-based spectroscopic technique potentially influenced by small shifts in room and samples temperature, being necessary a straight control of these parameters to ensure the reproducibility and repeatability of the technique and its validation for routine use.

\section{Conclusions}

In this work we demonstrate the ability of FT-NIRS to discriminate non-contaminated from bacteria contaminated saline solutions considering bacteria most frequently associated with contaminations in pharmaceutical products. Moreover, we also proved that this spectroscopic technique can be used to discriminate the saline solutions according to the contaminant bacteria and to quantify the extent of the contamination to very low concentrations. We further validated the method in commonly used pharmaceutical preparations. Additional work needs to be done in order to establish accurate calibration procedures to use this technique as routine; however, the results herein obtained highlight the potential of this technique as a reliable alternative to be used in the pharmaceutical industry for the detection and quantification of bacterial contaminations.

\section{Author's contibution}

CS and JL participated in the conception and design of the study. CQ, DM and CS contributed to data acquisition. CS and JL performed data analysis. CQ DM, JL, EF and CS contributed to data discussion and to the preparation of the manuscript. All the authors read and approved the final manuscript.

\section{Conflict of interest}

The authors have no competing interests.

\section{Acknowledgements}

This work was funded by Fundação para a Ciência e a Tecnologia (FCT) under the project UID/QUI/50006/2013. The authors also thank the FCT Strategic Project of UID/BIO/04469/2013 unit, the project RECI/BBB-EBI/0179/2012 (FCOMP-01-0124-FEDER-027462) and the project "BioInd-Biotechnology and Bioengineering for improved Industrial and Agro-Food processes", REF. NORTE-070124-FEDER-000028 co-funded by the Programa Operacional Regional do Norte (ON.2-O Novo Norte), QREN, FEDER. Daniela Mesquita (SFRH/BPD/82558/2011) and Cristina Quintelas were funded by post-doctoral grants from FCT and ON.2. Clara Sousa was funded by a CIENCIA2008 contract from FCT.

\section{Appendix A. Supplementary data}

Supplementary data associated with this article can be found, in the online version, at http://dx.doi.org/10.1016/j. ijpharm.2015.07.005.

\section{References}

Alsberg, B.K., Kell, D.B., Goodacre, R., 1998. Variable selection in discriminant partial least-squares analysis. Anal. Chem. 70, 4126-4133.

Anonymous, 2000. Evaluation, validation and implementation of new microbiological testing methods, Technical Report No. 33, PDA J. Pharmaceut. Sci. Tech. 54, 1-45.

Bakeev, K.A., 2005. Process Analytical Technology: Spectroscopy Tools and Implementation Strategies for the Chemical and Pharmaceutical Industries. Blackwell, Oxford.

Barker, M., Rayens, W., 2003. Partial least squares for discrimination. J. Chemom. 17, 166-173.

Blanco, M., Alcalá, M., Bautista, M., 2008. Pharmaceutical gel analysis by NIR spectroscopy: determination of the active principle and low concentration of preservatives. Eur. J. Pharm. Sci. 33, 409-414.

Buchbinder, L., et al., 1951. Studies to formulate new media for the standard plate count of dairy products. Public Health Rep. 66, 327-329.

Denyer, S.P., Baird, R.M., 2007. Guide to Microbiological Control in Pharmaceuticals and Medical Devices. CRC Press, Boca Raton, Florida.

European Pharmacopoeia. 8th edition (http://online.pheur.org/EN/entry.htm) (accessed 01.10.14.).

Geladi, P., Kowalsky, B.R., 1986. Partial least-squares regression: a tutorial. Anal. Chim. Acta 185, 1-17.

Hugo, W.B., Russell, A.D., 1998. Pharmaceutical Microbiology. Blackwell Scientific Publications, Oxford.

Hussaini, S.N., Hassanali, H.T., 1987. Limulus amoebocyte lysate assay of endotoxin: a method for visual detection of the positive gel reaction. J. Med. Microbiol. 24, 89-90.

Kricka, L.J., 1998. Prospects for chemiluminescent and bioluminescent immunoassay and nucleic acid assays in food testing and the pharmaceutical industry. J. Biolumin. Chemilumin. 13, 189-193.

Obuekwe, C.O., Obuekwe, I.F., Rafiq, M., 2000. Surface contamination in some common available dosage forms. Med. Princ. Pract. 9, 290-299.

Palou, A., et al., 2012. Determination of drug, excipients and coating distribution in pharmaceutical tablets using NIR-CI. J. Pharm. Anal. 2, 90-97.

Roda, A., Guardigli, M., 2012. Analytical chemiluminescence and bioluminescence: latest achievements and new horizons. Anal. Bioanal. Chem. 402, 69-76.

Rodriguez-Saona, L.E., Khambaty, F.M., Fry, F.S., Calvey, E.M., 2001. Rapid detection and identification of bacterial strains by Fourier transform-near infrared spectroscopy. J. Agric. Food Chem. 49, 574-579. 
Rodriguez-Saona, LE, Khambaty, F.M., Fry, F.S., Dubois, J., Calvey, E.M., 2004 Detection and identification of bacteria in a juice matrix with Fourier transform-near infrared spectroscopy and multivariate analysis. J. Food Prot. 67, 2555-2559.

Sarraguça, M.C., Lopes, J.A., 2009. Quality control of pharmaceuticals with NIR: from lab to process line. Vib. Spectrosc. 49, 204-210.

Savitzky, A., Golay, M.J.E., 1964. Smoothing and differentiation of data by simplified least squares procedures. Anal. Chem. 36, 1627-1639.
Sutton, S.V.W., Cundell, A.M., 2004. Microbial identification in the pharmaceutical industry. Pharm. Forum 30, 1884-1894.

Tito, N.B., Rodemann, T., Powell, S.M., 2012. Use of near infrared spectroscopy to predict microbial numbers on Atlantic salmon. Food Microbiol. 32, 431-436.

Tomuta, I., Rus, L., Iovanov, R., Rus, L.L., 2013. High-throughput NIR-chemometric methods for determination of drug content and pharmaceutical properties of indapamide tablets. J. Pharm. Biomed. Anal. 84, 285-292.

Wahl, P.R., et al., 2014. PAT for tableting: inline monitoring of API and excipients via NIR spectroscopy. Eur. J. Pharm. Biopharm. 87, 271-278. 